\title{
The Efficacy of Mandibular Advancement Appliances as a Treatment Alternative to Continuous Positive Airway Pressure in Moderate OSAHS Ama Johal' ${ }^{\text {* }}$, Preeti Jauhar', Fatemah Alqattan', Saba Kassim² and Kieran Mc Cloughlin'
}

${ }^{1}$ Oral Growth \& Development, Institute of Dentistry, Queen Mary University of London, UK

${ }^{2}$ Department of Preventive Dental Sciences, Taibah University Dental College \& Hospital, Al-Madinah Al-Munawwrah, Saudi Arabia

*Corresponding author: Ama Johal, Institute of Dentistry, Bart's and The London School of Medicine and Dentistry, Turner Street, London E1 2AD, UK, Tel: 0207-377-7686, Fax: 0207-377-7654, E-mail: a.s.johal@qmul.ac.uk

\begin{abstract}
Study objectives: Could a clinically safe and reliable compliance measure of mandibular advancement appliance (MAA) therapy demonstrate a high level of therapeutic efficacy, in patients diagnosed with moderate OSAHS.

Study design and participants: A long-term prospective observational study was undertaken in which patients with moderate OSAHS and in whom CPAP use had failed, to determine treatment compliance with MAA therapy, after a minimum period of 18 months, using an objective monitor. Treatment outcomes included both objective sleep monitoring and a determination of the therapeutic efficacy based on the calculation of the mean disease alleviation (MDA)

Results: Forty-two patients, with moderate OSAHS (mean AHI of 21.0 [+/- 10.3] events/hour) completed the study, at 18-month follow-up. A statistically significant difference $(p<0.001 ; 95 \% \mathrm{Cl} 2.76,3.50)$ was observed between the objectively recorded use of the MAA appliance (7.13 hours) and the predetermined gold standard level of 4 hours. A highly statistically significant reduction $(p<0.001)$ was observed in follow-up ESS (7.2 +/- 3.3) and AHI $(4.7+/-3.0)$ scores, which in turn was evidenced by the calculated therapeutic efficacy of $75.1 \%(95 \mathrm{Cl}$ $70.9,79.2)$ and a MDA of $70 \%(95 \mathrm{Cl} 63.7,76.3)$.

Conclusion: The study demonstrates the safe long-term objective measurement of compliance and comparable levels of therapeutic efficacy for MAA therapy in patients with moderate OSAHS providing new evidence of the role of MAA therapy as a viable alternative in patients unable to tolerate CPAP.
\end{abstract}

\section{Keywords}

Observational study, Mandibular appliances, Sleep apnoea, Compliance

\section{Brief Summary}

\section{Current knowledge/study rationale}

Mandibular advancement appliances are recognised first alternative therapy to CPAP. To-date no study has objectively measured MAA compliance and therefore therapeutic effect of this alternative treatment modality in moderate OSAHS patients.

\section{Study impact}

The present study demonstrates not only the long-term clinical safety of an objective measure of MAA compliance but also provides a measure of the therapeutic efficacy in patients with moderate OSAHS, who were unable to use CPAP therapy.

\section{Introduction}

Obstructive sleep apnoea hypopnoea syndrome (OSAHS) is a condition characterised by the repeated collapse of the pharyngeal airway, resulting in either complete (apnoea) or partial obstruction (hypopnoea) during sleep. The resultant sleep fragmentation, hypoxia and hypercapnia can have significant clinical consequences including excessive daytime sleepiness, increased cardiovascular effects and impaired cognitive functioning [1-3]. Davies and Stradling, [4] reviewed 12 major worldwide prevalence studies for obstructive sleep apnoea and suggested that once methodological and disease definition factors are considered, 1 to 5 per cent of adult men and fewer women have sleep apnoea syndrome.

Nasally applied continuous positive airways pressure (CPAP) is regarded as the major non-surgical, long-term treatment - the so-called 'Gold standard' for OSAHS, with significant reversal of symptoms whilst maintaining airway patency. Most patients require lifelong treatment and to be reliably effective, CPAP should be used for 4 to 6 hours per night, 7 days a week [5]. However, its main limitation remains patient compliance, with a recent study demonstrating the overall efficacy of the sub-optimally used CPAP being similar to surgical treatment [6]. Increasingly, the role of mandibular advancement appliances (MAA) therapy in the treatment of OSAHS is being recognised, with the American Academy of Sleep Medicine considering it as the first line alternative to CPAP in the management of mild-moderate OSA [7].

The ability to assess compliance accurately provides a true measure of a treatment modality's effectiveness. With CPAP therapy, the inbuilt compliance monitor has provided valuable insight into the limitations of self-reported use, with patients overestimating by up to 1 hour [8]. More recently, Vanderveken, et al. [9] and Dieltjens, et al. [10] reported on the safety and feasibility, at 3- \& 12- months respectively, of objective measurement of oral appliance use in the same cohort of patients, who demonstrated a range of sleepdisordered breathing, from snoring to OSA.

Importantly, if MAA are to be acknowledged as the first line alternative to CPAP, the ability to safely and reliably (objective)

Citation: Johal A, Jauhar P, Alqattan F, Kassim S, Mc Cloughlin K (2016) The Efficacy of Mandibular Advancement Appliances as a Treatment Alternative to Continuous Positive Airway Pressure in Moderate OSAHS. J Sleep Disord Manag 2:013 Copyright: (c) 2016 Johal A, et al. This is an open-access article distributed under the terms of the Creative Commons Attribution License, which permits unrestricted use, distribution, and reproduction in any medium, provided the original author and source are credited. 
measure MAA compliance, adds not only to our better understanding of this treatment, but perhaps of greater significance it provides a true measure of overall therapeutic efficacy, and in turn allows a better comparison with other treatment modalities. Whilst CPAP therapy remains more effective in controlling OSAHS, particularly severe OSAHS, it may be limited by its lower compliance. Long-term validation of the use and safety of objective measures of MAA therapy in patients, who have failed CPAP therapy, is needed. In addition, the theoretical proposal that MMA treatment could achieve an equivalent level of therapeutic efficacy to that achieved with CPAP therapy warrants further investigation, in patients with moderate OASHS [9]

The present study thus aimed to assess the long-term therapeutic efficacy of MAA therapy in patients with moderate OSAHS, who had failed CPAP therapy and clinical safety and reliability of the compliance monitor in use. We hypothesised that a more simplistic and clinically safe manner of attaching the compliance device to an MAA could be achieved for everyday use and that an equivalent level of therapeutic efficacy to CPAP therapy could be achieved in patients diagnosed with moderate OSAHS

\section{Methods}

\section{Study design and participants}

This prospective observational study was approved by the Regional Research and Ethics committee (2012/LO/0854). Participants referred for MAA therapy to a Specialist clinic within the Institute of Dentistry were consecutively recruited on the basis of meeting the following study selection criteria: Failed or were intolerant of CPAP therapy, a confirmed diagnosis of obstructive sleep apnoea hypopnoea syndrome (AHI $>5$ events per hour), an Epworth sleepiness scale score suggestive of daytime sleepiness $\left(>10^{1}\right)$ with optimal dental/ periodontal health and free of temporomandibular joint disease (TMJ). Objective and subjective compliance was assessed after each subject had been using their MAA for a minimum of 18 months, in order to determine its use longitudinally.

A sample size estimation was based on data from the review by Weaver and Sawyer, [11] designed to assess compliance with CPAP. Thus, considering the best reported outcome $(60 \%)$ and a power of $80 \%$, with an alpha of 0.05 , the estimated sample size for a one-sample comparison of proportion to the hypothesised value $(p=0.45)$ was 40 participants. An additional 4 participants were recruited to allow for a $10 \%$ drop-out rate.

\section{MAA and compliance measurement}

On the basis of the identified selection criteria, a total of 55 subjects were screened for eligibility, of which $11(20 \%)$ were initially judged unsuitable. One subject was edentulous, 1 subject suffered TM] dysfunction and the remaining 9 demonstrated poor dental/periodontal health. All participants were provided with a $3^{\text {rd }}$ generation customised MAA, the 'Medical Dental Sleep Appliance' (MDSA; R. J. and V. K. Bird, Middle Park, Victoria, Australia) by a dental specialist and self-adjusted for mandibular advancement, to a maximum of $9 \mathrm{~mm}$ in accordance with an established treatment protocol for titration, to achieve maximum therapeutic benefit [12-15].

Participants were initially diagnosed with OSAHS on the basis of a limited home sleep study (Edentrace, Eden Tec, Minnesota, and USA) and underwent a similar repeat sleep study, with their MAA in-situ, after a minimum period of 18 months of wear. Apnoea was defined as a cessation of airflow of more than 10 seconds duration. Hypopnoea was defined as a 50 per cent reduction in the flow signal or thoracoabdominal movement, lasting more than 10 seconds accompanied by a fall of $\geq 4$ per cent in oxygen saturation. The AHI was determined by dividing the total numbers of apnoeas and hyponoeas by the total sleep time. An experienced specialist technician undertook the scoring, blind to the study.

Subjective compliance with MAA use was simultaneously assessed, during a 2-week period, following a minimum of 18 months of MAA treatment with a sleep diary, issued by the sleep clinic.

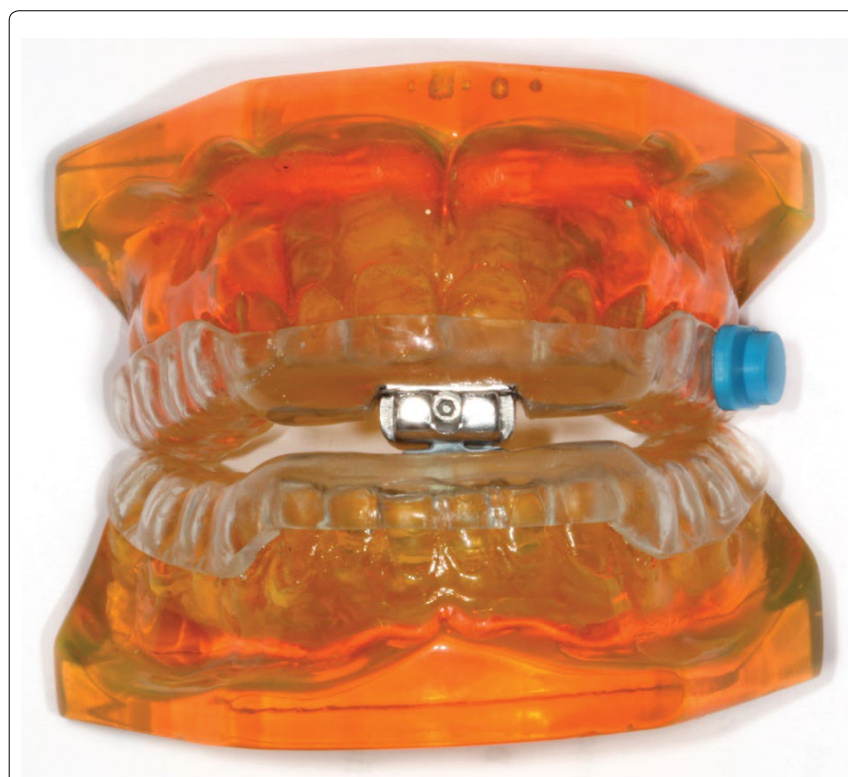

Figure 1: Thermo micro sensor (TheraMon ${ }^{\circledR}$, Handelsagentur Gschladt, Hargelsberg, Austria), used to measure objective compliance, attached to mandibular advancement appliance.

Participants were asked to record the following each night: time of appliance insertion and removal; bedtime and awake time, from which total sleep time (TST) was determined. In addition, the diary recorded the number of awakenings per night.

Objective compliance recording was determined using a thermo micro sensor (TheraMon ${ }^{\circ}$, Handelsagentur Gschladt, Hargelsberg, Austria), designed to record temperatures in excess of $35{ }^{\circ} \mathrm{C}$ (Figure 1). MAA compliance was then based on the assumption that when the thermometer exceeded $35{ }^{\circ} \mathrm{C}$, consistent with intra-oral mouth temperature, the splint was being worn. A novel method was developed to facilitate easy chair side attachment of the micro sensor to the MAA, in which $0.7 \mathrm{~mm}$ thermo-formed template (Essix, Ortho-care, West Yorkshire, UK) encasements were laboratory fabricated using a 'dummy' micro sensor in advance. The actual micro sensor was then simply placed in the template and fixed to the MAA using a cold-cure resin (Forestacryl, Forestadent, Buckingham shire, UK). The sensor was set to record temperature at 15 minutes intervals and the data was saved to its integral memory. Data was subsequently transmitted and analysed, using radiofrequency identification technology to TheraMon ${ }^{\star}$ Reader Station software (TheraMon ${ }^{\circledR}$ software, version 2.1) [16]

In using the TheraMon ${ }^{\oplus}$ micro sensor, a number of clinical safety endpoints were defined and recorded on a serious adverse event report form. These were: detachment of the micro sensor from the MAA; signs or symptoms of oral mucosal irritation or burns and any reported interference with MAA wear. In addition, the study endpoint was defined as the successful data capture and graphical display of compliance from the micro sensor.

\section{Additional measures}

MAA outcome questionnaire: In addition to all participants undergoing an objective follow-up sleep study with their MAA in$s i t u$, a questionnaire-based evaluation was also undertaken, designed to assess MAA compliance, side-effects and symptom change using a 10-point visual analogue scale (VAS). In addition, baseline and 18-month follow-up Epworth sleepiness scale questionnaires were used to subjectively record daytime sleepiness.

\section{Data and statistical analysis}

Data were analysed using the SPSS (Version 17.0, New York, USA), with significance taken at the 0.05 level. Descriptive statistics were used for participant demographics and data from the MAA outcome questionnaire. The Shapiro-Wilks test was used to assess 


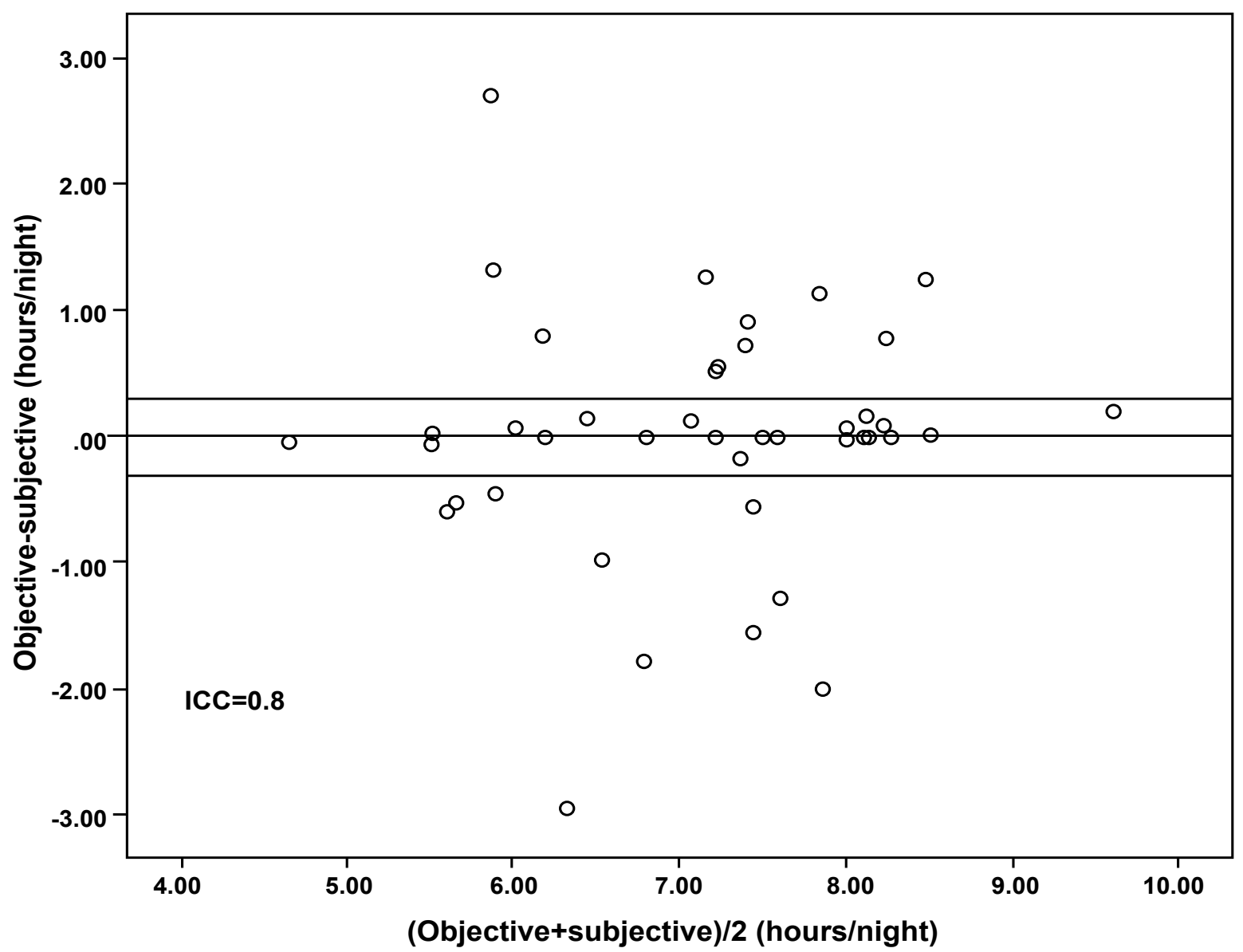

Figure 2: Bland-Altman plot, with $95 \%$ confidence intervals and calculated Intra-class correlation coefficient between subjective and objective compliance at 18 months.

Table 1: Baseline demographics of subjects participating in the study $(n=42)$.

\begin{tabular}{|l|l|}
\hline Variable & Mean (SD) Median (range) or $\mathbf{n}(\%)$ \\
\hline Age (years) & $56.1(10.7)$ \\
\hline Gender: & \multicolumn{2}{|l|}{} \\
\hline Males & $30(71)$ \\
\hline Females & $12(29)$ \\
\hline Neck circumference $(\mathrm{cm})$ & $40(2.9)$ \\
\hline Body mass index $\left(\mathrm{Kg} / \mathrm{m}^{2}\right)$ & $27.9(3.6)$ \\
\hline Apnoea-hypopnoea index (events/hour) & $21.0(10.3)$ \\
\hline Oxygen desaturation index (events/hour) & $11.0(3-16)$ \\
\hline Mean saturation $(\%)$ & $95.0(1.3)$ \\
\hline Minimum oxygen saturation (\%) & $85.1(6.2)$ \\
\hline Epworth sleepiness scale & $11.8(5.1)$ \\
\hline
\end{tabular}

normality of distribution. A one-sample t-test assessed for the level of compliance with the 'gold standard' of 4 hours. Student's t-test was used to assess the difference between subjective and objective compliance, change in the AHI and ESS values. Therapeutic efficacy was calculated (AHI baseline minus AHI with MAA in-situ, divided by the AHI baseline) and expressed as a percentage [10]. Furthermore, both the adjusted MAA compliance (objective MAA compliance divided by total sleep time) and mean disease (OSA) alleviation (adjusted MAA compliance $\times$ therapeutic efficacy $\div 100$ ), expressed as a percentage, were calculated as recommended by Grote, et al. [17] and Vanderveken, et al. [9] Results are expressed as mean +/- standard deviation (SD) or as number (\%) of participants.

\section{Results}

A total of 42 of 44 participants (30 male) completed the 18-month observational study period ( $96 \%$ of the total sample; (Table 1). Two participants were lost to follow-up as a result of work relocation. The mean age of participants was $56(+/-10.7)$ years. Participants were overweight, with a mean body mass index of $27.9(+/-3.6) \mathrm{Kg} / \mathrm{m}^{2}$ and neck circumference of $40(+/-3.0) \mathrm{cm}$. Participants demonstrated a moderate degree of obstructive sleep apnoea-hypopnoea syndrome, with a mean AHI of $21(+/-10.3)$ events per hour \& Epworth sleepiness scale score of $11.8(+/-5)$.

\section{Objective monitor clinical safety}

Successful attachment of the micro-sensor was achieved with all MAA devices. The defined clinical safety end point was achieved in all 42 participants, with no adverse events being recorded.

\section{MAA compliance measures}

A statistically significant difference $(\mathrm{p}<0.001 ; 95 \%$ CI 2.76 , 3.50) was observed between the objectively recorded use of the MAA appliance (7.13 hours) and the predetermined gold standard level (4 hours), with 93 per cent exceeding this gold standard. The mean (SD) hours of reported and objective wear were $7.38(+/-0.90$ range 5.36 to 9.54$)$ and 7.13 (+/- 1.19 range 4.63 to 9.71$)$ respectively, per night. Whilst the difference ( 0.24 minutes) reached statistical significance ( $\mathrm{p}=00.1$ ), with self-reported hours of wear being higher, intra-class correlation coefficient (0.8) demonstrated a high level of agreement between the two reported measures of wear (Figure 2), with a Cronbach alpha value of 0.9 (95\% CI, 0.78-0.94). This translated to a $93.2(95 \mathrm{CI} 87.5,98.9)$ per cent adjusted MAA compliance.

\section{MAA efficacy}

A highly statistically significant reduction $(\mathrm{p}<0.001)$ was observed in follow-up ESS $(7.2+/$ - 3.3) and AHI $(4.7+/$ - 3.0) scores, with an eta squared statistic $(0.6 \& 0.8$, respectively) indicating a large impact size for MAA therapy (Table 2), with $95 \%$ and $67 \%$ of subjects achieving an AHI of less than 10/hour and 5/hour, respectively. (Figure 3) further demonstrates the heterogeneity in AHI response to 


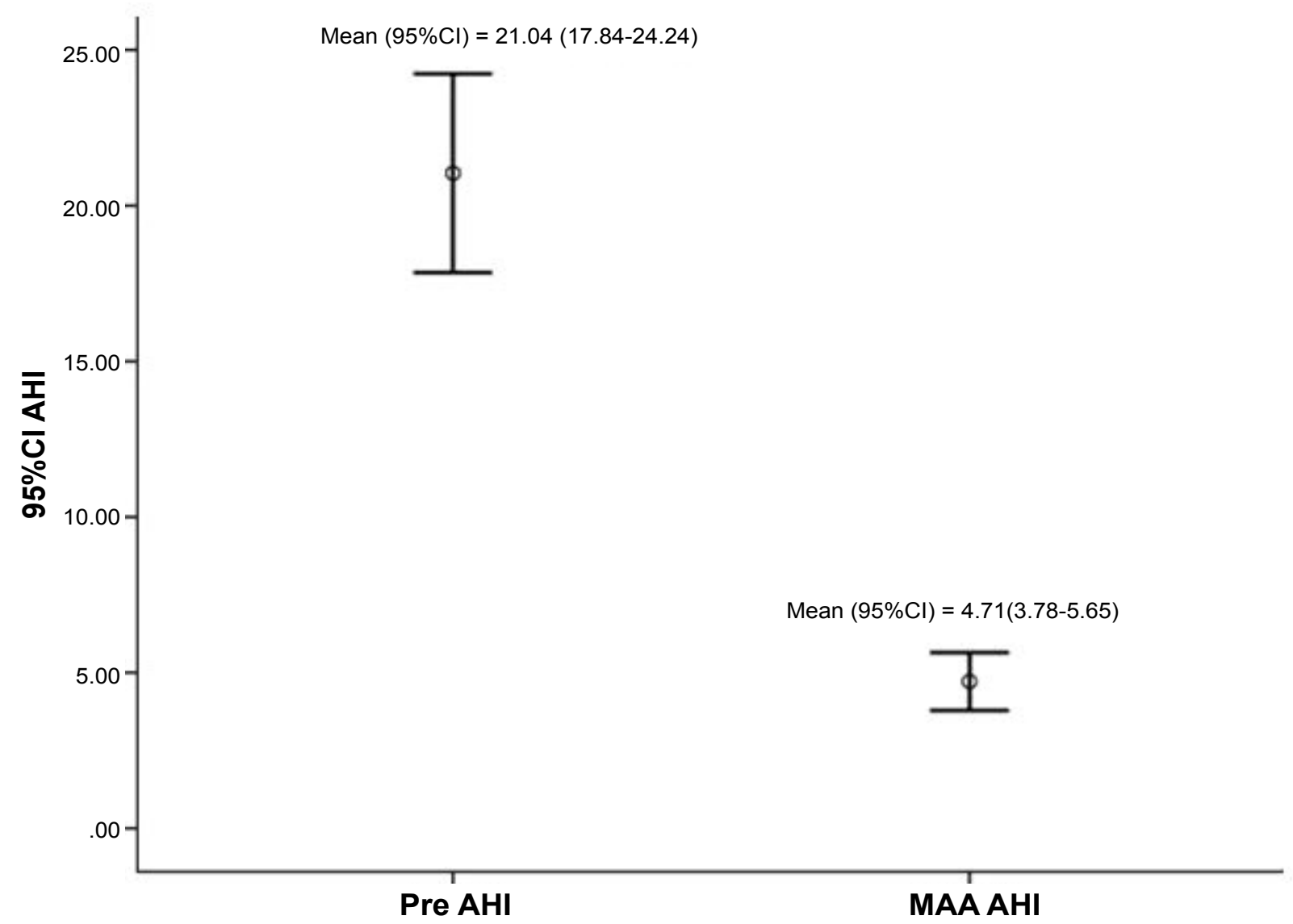

Figure 3: Box plots demonstrating the baseline and mandibular advancement appliance (MAA) apnoea-hypopnoea index (AHI) scores and 95\% confidence intervals $(\mathrm{Cl})$.

Table 2: Treatment outcomes with mandibular advancement appliance (MAA) therapy in relation to objective and subjective measures $(n=42)$. Measures are reported as mean (SD) or number (\%).

\begin{tabular}{|l|l|l|l|}
\hline Variable & Pre-treatment & Post-treatment & p Value \\
\hline $\begin{array}{l}\text { Apnoea-hypopnoea index } \\
\text { (events/hour) }\end{array}$ & $21.0(10.3)$ & $4.7(3.0)$ & $p<0.001$ \\
\hline Therapeutic efficacy (\%) & & 75.1 & \\
\hline Mean disease alleviation (\%)** & & 70.0 & \\
\hline Epworth sleepiness scale & $11.8(5.1)$ & $7.2(3.3)$ & $\mathrm{p}<0.001$ \\
\hline
\end{tabular}

*Therapeutic efficiency (\%) was calculated as follows: $\mathrm{AHI}$ baseline minus $\mathrm{AHI}$ with MAA in-situ, divided by the AHI baseline.

${ }^{* *}$ Mean disease alleviation (\%) was calculated as follows: adjusted MAA compliance $\times$ therapeutic efficacy $\div 100$.

treatment. This was evidenced by the calculated therapeutic efficacy of 75.1 (95CI 70.9, 79.2) per cent and a mean disease alleviation (MDA) of 70 (95CI 63.7, 76.3) per cent (Figure 4).

Participants reported a mean VAS effectiveness score for their MAA of $8.6(+/-1.8)$, with the majority $(93 \%)$ rating the appliance effectiveness as $\geq 5$ and 64 per cent rating the MAA as being $\geq 9$. With regards to the effect of the appliance on their quality of sleep, the mean VAS score was $8.0(+/-1.9)$, indicating a significant improvement. The questionnaire also assessed the reasons, if any for removal of their MAA during the night, with participants reporting this related to: going to the toilet $(n=8)$, thirst $\&$ the need to drink ( $\mathrm{n}$ $=8)$, dental discomfort $(n=5)$ and discomfort of the jaw $(n=3)$. All patients reported titration of their MAA, this ranged from 5-9 $\mathrm{mm}$.

\section{Discussion}

The present research significantly adds to the knowledge-base on the role of MAA therapy as an alternative treatment the management of OASHS patients, who failed CPAP treatment. The particular strengths of this study include the ability to now report on long-term objective compliance in patients fitted with MAA, using a simple and reliable method which has been pioneered in our centre. The favourable compliance observed with a customised MAA has resulted in a similar adjusted AHI and clinical efficacy to that cited in the literature for CPAP therapy in OSAHS. This in turn has important implications for patient whose sleepiness must be monitored for medico-legal reasons, such as driving ability. Additionally, the development of a novel compliance monitor for MAA use will add vastly to the efficacy with which future randomised, controlled trials between devices are conducted.

The current non-surgical management of patients with OSAHS relies on life-long treatment, which is entirely patient-dependent. Thus, the importance of being able to safely and reliably record compliance in an objective manner becomes apparent. In this context, either CPAP or MAA use alone does not necessarily translate to treatment efficacy, whereas a measure of mean OSA (disease) alleviation (MDA) not only serves to provide a measure of therapeutic efficacy but also adds greater meaningfulness to comparison between these two different treatment modalities by taking account of objective compliance $[9,10,18]$. The importance of evaluating this criterion is evident in relation to measuring how much treatment is sufficient to control the symptoms of OASHS and related cardiovascular risk factors, along with driving performance. Grote, et al. [17] in a retrospective study demonstrated only a 50 per cent long-term clinical effectiveness of CPAP therapy, largely attributable to the low (50\%) acceptance. With the acknowledged role of MAA therapy as both a first line treatment in mild-moderate OSAHS and its principle role as the alternative therapy in patients unable to cope with or refusing CPAP [7] the need for their long-term assessment of therapeutic efficacy becomes extremely important. More recently, a number of studies $[19,20]$ have reported the importance of differences in efficacy and compliance between CPAP and MAA are likely to influence improvements in health outcomes, with the reported greater efficacy of CPAP being offset by its inferior compliance to MAA. The authors suggested that long-term follow-up, with objective measurement of 


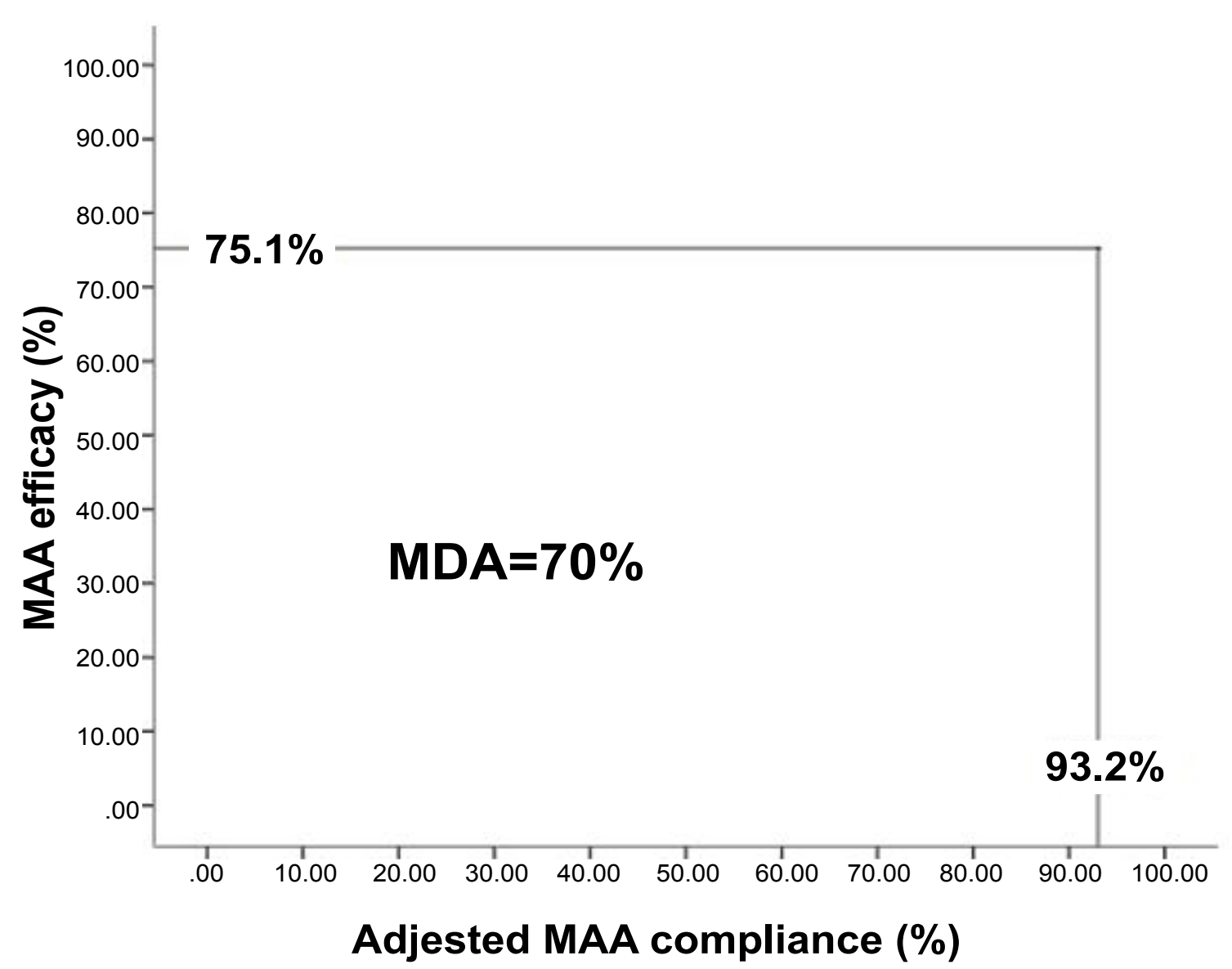

Figure 4: The mean disease alleviation (MDA) provided a measure of the overall therapeutic effectiveness (\%) of the mandibular advancement appliance (MAA) and was determined from the adjusted MAA compliance and therapeutic efficacy.

MAA compliance was needed to better define treatment strategies in OSA [19,20]. Similarly,[21] in their 2 year follow-up of MAA versus CPAP suggested the former could serve as a long-term alternative in patients with moderate to severe OSAHS, in whom CPAP treatment fails. However, they remained reliant on self-reported MAA use, acknowledging the need for objective measure.

Whilst CPAP therapy has benefited from the inclusion of an objective compliance measure, this remains new to the field of MAA therapy, and only a single cohort of patients with a range of sleep related breathing disorders being successfully reported $[9,10]$ Importantly, no previous evaluation of compliance measure has been performed in patients with moderate OSAHS, who have failed CPAP therapy. Previous attempts to develop an objective measure of compliance have proven highly unsuccessful due to a range of technical problems, which precluded their practicality of use in everyday clinical practice [22,23]. The present study supported the previous findings in relation to both safety and reliability of the TheraMon ${ }^{\star}$ micro sensor to objectively record MAA compliance, with no adverse events being recorded [9,10]. Furthermore, a new novel method for facilitating attachment, without affecting either the MAA fit or performance of the micro-sensor was developed.

An objective measure of MAA compliance permitted the mean OSA alleviation (MDA) to be calculated as an expression of therapeutic efficacy. An MDA value of 70 per cent was calculated in the present study at 18 months and compares very favourably with both the previously reported MAA value of 54.9 per cent, at 1 year [10] and the CPAP value of 52.4 per cent, at 30 months $[6,17]$ Despite the fact that CPAP therapy can achieve better reduction in the AHI, this data supports the original hypothesis that the higher levels of compliance achieved with MAA treatment translate into a comparable or better therapeutic effectiveness reported in the literature with CPAP therapy $[6,9,19,20]$. The more favourable long-term results of MAA treatment observed in the present study combine a high adjusted compliance (93.2\%) with an equally high therapeutic efficacy (75.1\%). This in turn reflects the highly significant improvements observed in both objective (AHI and ESS) measures and reported subjective symptoms. In contrast, the reported overall lower therapeutic efficacy (54.9) reported by Dieltjens, et al. [10] could be explained by the selection of the MAA, with the present study using an optimally designed and retentive customised MAA, permitting not only incremental $(0.5 \mathrm{~mm})$ mandibular advancement in the sagittal plane, in line with a well- established titration protocol but also lateral translation and minimal vertical opening during sleep $[10,13-15,23]$.

The present study also provided a measure of long-term MAA compliance, with a 96 per cent response rate observed in this observational study of participants with moderate OSAHS. This was supported by the observed adjusted compliance rate and compares favourably with the discontinuation rate of 9.8 per cent reported by Dieltjens, et al. [10] in their 1 year follow-up of patients with a range of sleep disordered breathing, from mild to severe OASHS Thus the present study supports the concept that MAA therapy has a high patient acceptance and preference, reflected in the lower discontinuation rate compared with CPAP [24]. In stark contrast, 20-50 per cent of patients have discontinued CPAP therapy at 1 year follow-up [25].

The present study is not without its limitations. These include the relatively small sample being reported. However the power of the study was maintained by inflating the recruitment numbers by 10 percent, at commencement. In addition, patients were asked to subjectively record their time to bed, wake-up and hours of sleep to determine the total sleep time. However, the present study attempted to overcome previously reported limitations of applying a retrospective questionnaire to assess self-reported wear, by the use of a prospective diary on self-reported use [10]. The generalizability of the findings may be limited by the fact that the Centre specialises 
in the management of MAA therapy in OASHS patients and as such may have achieved a more optimal treatment outcome and as such there is the potential for both selection and performance bias. This may also partly explain the very high observed continuation rate with MAA treatment, as patients are followed up every 6 months and are given ready access to professional support if any problems are encountered with their MAA. Furthermore, the patients in this cohort, were all referred and provided MAA treatment on the basis of failed CPAP therapy \& demonstrated treatment need. As such, the present study focussed on the specific guidance of MAA therapy being acknowledged as the first alternative to CPAP treatment, in order to provide a meaningful addition to the evidence-base for this practice. The risk of patients experiencing the Hawthorn effect in the absence of a randomised controlled trial (RCT) cannot be out ruled. However, all subjects were consecutively recruited following their initial referral and the study evaluated the outcome longitudinally, at 18 months, in an attempt minimise this effect [26]. A RCT, comparing MAA and CPAP therapy in symptomatic patients with moderate OSAHS could have been considered unethical, with the established evidencebase of CPAP being the Gold standard, first line, treatment in such individuals.

The present study demonstrates the safe long-term objective measurement of compliance and comparable levels of therapeutic efficacy for MAA therapy in patients with moderate OSAHS, providing new evidence of the role of MAA therapy as a viable alternative in patients unable to tolerate CPAP.

\section{Ethical Statement}

This prospective observational study was approved by the Regional Research and Ethics committee (2012/LO/0854). All patients gave written informed consent.

\section{Conflicts of Interest to Declare}

There are none in relation to any author.

\section{Financial Disclosure}

A small charities grant was awarded to help purchase the objective monitoring sensors.

\section{References}

1. Johns MW (1993) Daytime sleepiness, snoring, and obstructive sleep apnoea. The Epworth Sleepiness Scale. Chest 103: 30-36.

2. Horne J, Reyner L (1995) Sleep related vehicle accidents. Brit Med J 310 565-567

3. Lavie P, Herer P, Hoffstein V (2008) Obstructive sleep apnoea syndrome as a risk factor for hypertension: a population study. Brit Med J 320: 479-482.

4. Davies RJ, Stradling JR (1995) The epidemiology of sleep apnea. Am Rev Respir Crit Care Med 152: 711-716

5. Engleman HM, Martin SE, Kingshott RN, Mackay TW, Deary IJ, et al. (1998) Randomised placebo controlled trial of daytime function after continuous positive airway pressure (CPAP) therapy for the sleep apnoea/hypopnoea syndrome. Thorax 53: 341-345.

6. Ravesloot MJ, de Vries, N (2011) Reliable calculation of the efficacy of nonsurgical and surgical treatment of obstructive sleep apnea revisited. Sleep 34: $105-110$
7. Ramar K, Dort LC, Katz SG, Lettieri CJ, Harrod CG, et al. (2015) Clinical Practice Guidelines for the treatment of obstructive sleep apnoea and snoring with oral appliance therapy: An update for 2015. Journal of Clinical Sleep Medicine 11: 773-827.

8. Kribbs NB, Pack AI, Kline LR, Smith PL, Schwartz AR, et al. (1993) Objective measurement of patterns of nasal CPAP use by patients with obstructive sleep apnea. Am Rev Respir Dis 147: 887-895.

9. Vanderveken OM, Dieltjens M, Wouters K, De Backer WA, Van de Heyning $\mathrm{PH}$, et al. (2013) Objective measurement of compliance during oral appliance therapy for sleep-disordered breathing. Thorax 68: 91-96.

10. Dieltjens M, Braem MJ, Vroegop AV, Wouters K, Verbraecken JA, et al. (2013) Objectively measured vs self-reported compliance during oral appliance therapy during oral appliance therapy for sleep-disordered breathing. Chest 144: $1495-1502$

11. Weaver TE, Sawyer AM (2010) Adherence to continuous positive airway pressure treatment for obstructive sleep apnoea: implications for future interventions. Indian J Med Res 131: 245-258.

12. Barnes M, Mc Evoy RD, Banks S, Tarquinio N, Murray CG, et al. (2004) Efficacy of positive airway pressure and oral appliance in mild to moderate obstructive sleep apnea. Am J Respir Crit Care Med 170: 656-664.

13. Johal A (2008) A review of the use of mandibular advancement appliances in sleep-disordered breathing. Dent Update 35: 230-235.

14. Tsuiki S, Lowe AA, Almeida FR, John A Fleetham, et al. (2004) Effects of an anteriorly titrated mandibular position on awake airway and obstructive sleep apnea severity. Am J Orthod Dento facial Orthop 125: 548-555.

15. Gao X, Otsuka R, Ono T, Honda E, Sasaki T, et al. (2004) Effect of titrated mandibular advancement and jaw opening on the upper airway in nonapneic men: A magnetic resonance imaging and cephalometric study. Am J Orthod Dentofacial Orthop 125: 191-199.

16. McLaughlin K, Jauhar $P$, Johal A (2014) Thermo-processed dental adaptation and placement of an intraoral micro-sensor for biomedical research purposes: a new technique. Technologist 33: 8-11.

17. Grote L, Hedner J, Grunstein R, Kraiczi H (2000) Therapy with nCPAP incomplete elimination of sleep related breathing disorder. Eur Respir J 16: 921-927.

18. Lowe AA, Sjoholm TT, Ryan CF, Fleetham JA, Ferguson KA, et al. (2000) Treatment, airway and compliance effects of a titratable oral appliance. Sleep 23: S172-178.

19. Phillips CL, Grunstein RR, Darendelilier MA, et al. (2014) Health outcomes of continuous positive airway pressure versus oral appliance treatment for obstructive sleep apnea. Am J RespirCrit Care Med 187: 879-887.

20. Dal-Fabbro C, Garbuio S, D Almeida V, Cintra FD, Tufik S, et al. (2014) Mandibular advancement device and CPAP upon cardiovascular parameters in OSA. Sleep Breath 18: 749-759.

21. Doff MH, Hoekema A, Wijkstra PJ, van der Hoeven JH, Huddleston Slater JJ, et al. (2013) Oral appliance versus continuous positive airway pressure in obstructive sleep apnea syndrome: A 2-year follow-up. Sleep 36: 1289-1296.

22. Inoko Y, Yoshimura K, Kato C, Osami Mortia, Masaki Kohno (2009) Efficacy and safety of temperature data loggers in measuring compliance with the use of oral appliances. Sleep Biol Rhythms 7: 188-192.

23. Dieltjens M, Vanderveken OM, Heyning PH, Braem MJ (2012) Current opinions and clinical practice in the titration of oral appliances in the treatment of sleep-disordered breathing. Sleep Med Rev 16: 177-185.

24. Ferguson KA, Ono T, Lowe AA, Keenan SP, Fleetham JA, et al. (1996) A randomised crossover study of an oral appliance vs nasal-continuous positive airway pressure in the treatment of mild-moderate obstructive sleep apnoea. Chest 109: 1269-1275.

25. Engleman HM, Wild MR (2003) Improving CPAP use by patients with the sleep apnoea/hypopnoea syndrome (SAHS). Sleep Med Rev 7: 81-99.

26. Adair, JG (1984) The Hawthorne effect: A reconsideration of the methodological artifact. J Appl Psychology 69: 334-335. 